\title{
THE ISSUES OF HOUSING THE ELDERLY WITH THEIR COMPANION ANIMALS IN CARE HOMES
}

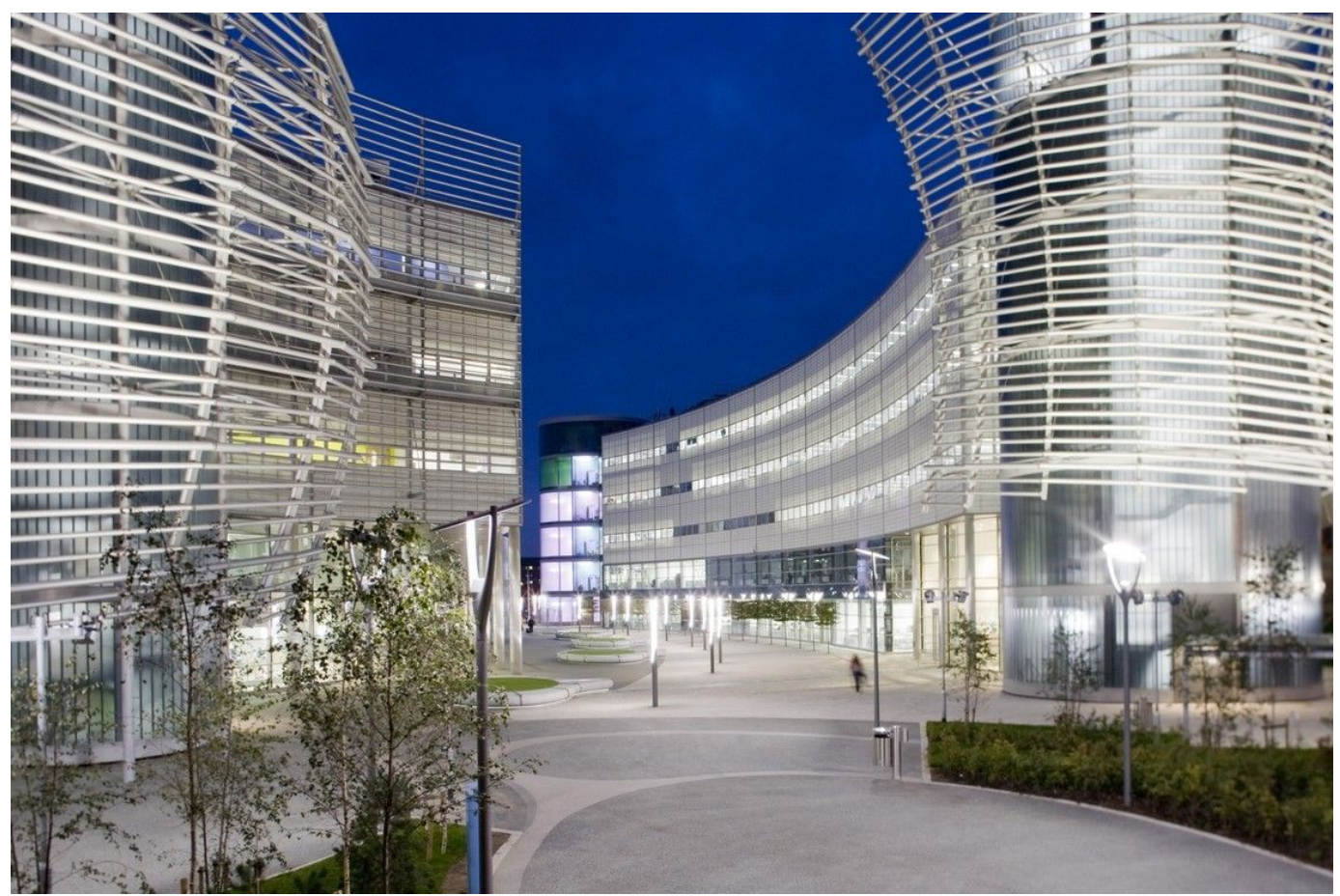

Student Law Office, Policy Clinic, Northumbria University, School of Law

Research report for UK Centre for Animal Law (ALAW) undertaken by Marija Bilerte, Golara Bozorg and Amy Millross. Supervised by Dr Rachel Dunn.

May 2019 


\section{Table of Contents}

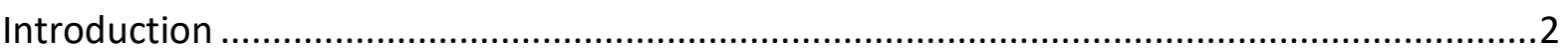

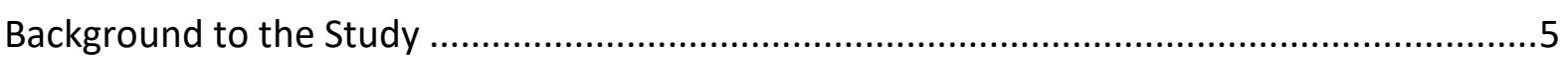

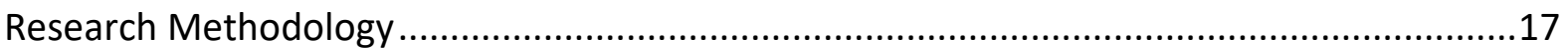

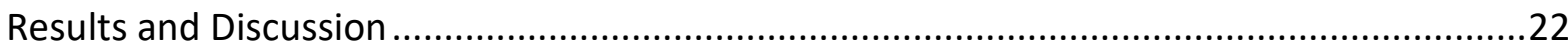

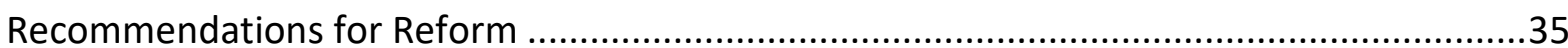

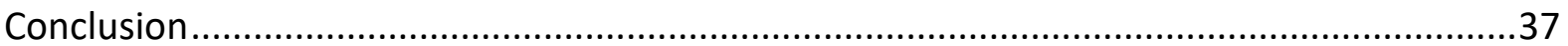

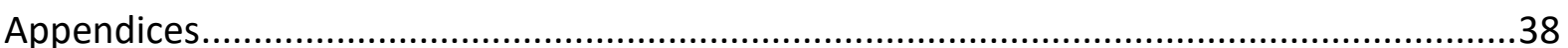




\section{Introduction}

Our team was appointed by Ms Paula Sparks, from the UK Centre for Animal Law (ALAW), to carry out research into the difficulties for the elderly population and their companion animals to remain living together in supported accommodation/care homes. As stated on their website $^{1}$, A-law is a charity which brings together lawyers and others interested in animal protection law, to share experience and to harness that expertise for the benefit of animals, by securing more comprehensive and effective laws and better enforcement of existing animal protection laws. In the UK, they are a unique organisation, focusing on promoting knowledge and understanding - both amongst lawyers, universities, animal protection groups and the wider public - about animal protection laws in the UK and elsewhere around the world.

We have conducted this report to find out about the prevalence of difficulties faced by the elderly population in Newcastle upon Tyne to find suitable accommodation with their companion animal. We looked into the reasons why the elderly people with companion animals are unable to access certain accommodations with their companion animals and whether this is due to legal and/or regulatory factors or perception of the same or other reasons.

We began our research by exploring the reasons why companion animals should be able to accompany their humans into care homes, whether there are benefits in doing so and what impacts, if any, they may have on the humans in general and on the elderly population in particular. This was done through a review of the literature. In order to have a better understanding of the situation, we decided to conduct interviews with the managers of a number of care homes in the North East of England, to find out more about the issue in hand, and the reasons they give for either allowing all kinds of pets, allowing certain kinds of pets, or not allowing pets at all.

We wished to find out whether there are any steps that could be taken to reduce the obstacles faced by the elderly owners of companion animals who are entering the care system and want to have their pets with them in their new accommodation. Further, we explored what steps are necessary to overcome those legal/regulatory obstacles, e.g. legislation, amendments and/or what steps would need to be taken to overcome perceived legal or regulatory obstacles (e.g. education or guidelines).

\section{Definitions}

\section{Companion Animal}

Coined by the animal welfare and veterinarian sector, it refers to the co-dependent relationship of humans and other animals and is variously defined as "mutuality of the human-animal relationship". ${ }^{2}$ It refers to "any domesticated, domestic-bred or wild-caught animals, permanently living in a community and kept by people for company, amusement,

\footnotetext{
${ }^{1}$ A-Law - UK Centre For Animal Law (Alaw.org.uk, 2019) <https://www.alaw.org.uk> accessed $1^{\text {st }}$ April 2019

2 Froma Walsh, 'Human-Animal Bonds I: The Relational Significance of Companion Animals' (2009) 48 Family Process
} 
work (e.g. support for blind or deaf people, police or military dogs) or psychological support including dogs, cats, horses, rabbits, ferrets, guinea pigs, reptiles, birds and ornamental fish". ${ }^{3}$

\section{Elderly Population}

In the North East of England, $18.5 \%$ of the population $(2,618,700)$ were aged 65 or older in 2014. ${ }^{4}$ The World Health Organisation has established that 'Most developed world countries have accepted the chronological age of 65 years as a definition of 'elderly' or older person'. 5 In England and Wales, there were 421,100 elderly care home residents over the age of 65 in $2017 .^{6}$

\section{Care Home}

According to the NHS website "a Care Home is a place where personal care and accommodation are provided together" 7 ; it is a residential setting where a number of older people live, usually in single rooms, and have access to on-site care services. A home registered simply as a care home will provide personal care - help with washing, dressing and giving medication. Some care homes are registered to meet a specific care need, for example dementia or terminal illness. ${ }^{8}$

In this report we refer to care homes and supported accommodation interchangeably and do not distinguish between them.

\section{Background}

For many people, pets are like family members. Companion animals can play a significant role in the life of all members of the family, especially in older family members' lives. With the improvement of medicine, we are facing an ageing population, many of whom, inevitably, have to move to care homes, nursing homes and such kind. Since many care homes through their policies restrict the allowance of pets (even those that often do, have strict restrictions), the elderly is faced with the dilemma of either leaving the loved family pets behind, or choose the animal over support and care in their old age. ${ }^{9}$

${ }^{3}$ CALLISTO, 'Final Report' (CORDIS 2014) <https://cordis.europa.eu/docs/results/289/289316/final1-callistoproject-final-report.pdf $>$ accessed $5^{\text {th }}$ November 2018

${ }^{4}$ Jonathan McMullan, 'Population Estimates For UK, England And Wales, Scotland And Northern Ireland: Office

For National Statistics' (Ons.gov.uk, 2019)

<https://www.ons.gov.uk/peoplepopulationandcommunity/populationandmigration/populationestimates/bul letins/annualmidyearpopulationestimates/mid2017/previous/v1> accessed $1^{\text {st }}$ April 2019

${ }^{5}$ World Health Organisation, 'Proposed Working Definition Of An Older Person In Africa For The MDS Project' (World Health Organization, 2018) <http://www.who.int/healthinfo/survey/ageingdefnolder/en/> accessed $12^{\text {th }}$ November 2018

${ }^{6}$ Laing and Buisson, Care of Older People UK Market Report (2017) <https://www.laingbuisson.com/wpcontent/uploads/2016/06/Care OlderPeople 27ed Bro WEB.pdf> accessed 30 ${ }^{\text {th }}$ April 2019

7 'Supporting Information: Care Home' (Datadictionary.nhs.uk, 2019)

<https://www.datadictionary.nhs.uk/data_dictionary/nhs_business_definitions/c/care_home_de.asp?shownav=> accessed 30 ${ }^{\text {th }}$ April 2019.

8 'Residential Care Home (Definition Of)' (Housingcare.org, 2019) <http://www.housingcare.org/jargonresidential-care-homes.aspx $>$ accessed $30^{\text {th }}$ April 2019

${ }^{9}$ Deborah Rook, 'For The Love Of Darcie: Recognising The Human-Companion Animal Relationship In Housing Law And Policy' (2018) 39 Liverpool Law Review 29 
Unfortunately, when older people are left with no other option than moving into residential care, pets more often than not (especially cats and dogs) are not allowed to accompany them. ${ }^{10}$ We know that pets can be extremely important to people and their wellbeing, yet the lack of pet-friendly care homes prevent the elderly from keeping a pet once they move into care homes. This is something discussed in depth in the background research conducted. This would lead them to have a difficult time to find a new suitable home.

\section{$\underline{\text { Aims }}$}

The aims of the research, set by ALAW, were to identify:

- The prevalence of difficulties faced by the elderly population within Newcastle upon Tyne to find accommodation (whether sheltered or in care homes) with their companion animal;

- the reasons why elderly people with companion animals are unable to access accommodation with their companion animals and whether this is due to legal and/or regulatory factors or a perception of the same or other reasons.

- Any steps that could be taken to reduce the obstacles faced by elderly owners of companion animals who are entering the care system or need for sheltered accommodation.

Our focus was on care homes in Newcastle upon Tyne. We contacted a number of managers and asked them to participate in voluntary interviews, to help us understand what their care home pet policies are and the reasons that they have decided to adopt those policies. This way, we could directly collect some useful data which would enable us to examine the barriers faced by the pet loving elderly. We decided that semi-structured interviews would be best to achieve our objectives, as it would allow us structure, yet be flexible to peruse interesting ideas and opinions when needed.

This report displays the findings from this research project and recommends ways in which to improve the law on pets in care homes.

\section{We would like to thank UK Centre for Animal Law and Debbie Rook for working with us on this research.}

\footnotetext{
${ }^{10}$ Smith, R., Johnson, J., \& Rolph, S. 'People, pets and care homes: a story of ambivalence' (2011) 12(4) Quality in Ageing and Older Adults 217
} 


\section{Background to the Study}

\section{The Law}

Initial research found that there is a deficit of any law to deny companion animals in care homes and/or sheltered accommodation. Thus, it is at the discretion of a landlord, or those in charge of a property, to decide whether or not to include a 'no pet' covenant or clause in their contract. This can affect the relationship we have with companion animals and effects those from all areas of the country, regardless of gender, age or ethnicity. ${ }^{11}$ In care homes, however, the issue and lack of legislation is particularly prevalent, as the elderly are faced with the choice of either giving up their pet or moving into accommodation which has the care they need.

The Animal Welfare Act 2006 is the principal legislation for animal welfare in England and Wales. Under the Animal Welfare Act 2006, powers exist for secondary legislation and codes of practice to be made to promote the welfare of animals. ${ }^{12}$

The particular requirements of ensuring an animal's welfare is set out in S.9 of the Act $^{13}$ :

S.9 - Duty of person responsible for animal to ensure welfare:

(1) A person commits an offence if he does not take such steps as are reasonable in all the circumstances to ensure that the needs of an animal for which he is responsible are met to the extent required by good practice.

(2) For the purposes of this Act, an animal's needs shall be taken to include-

(a) its need for a suitable environment,

(b) its need for a suitable diet,

(c) its need to be able to exhibit normal behaviour patterns,

(d) any need it has to be housed with, or apart from, other animals, and

(e) its need to be protected from pain, suffering, injury and disease.

(3) The circumstances to which it is relevant to have regard when applying subsection (1) include, in particular-

(a)any lawful purpose for which the animal is kept, and

(b) any lawful activity undertaken in relation to the animal.

(4) Nothing in this section applies to the destruction of an animal in an appropriate and humane manner.

The above duties are ones which a pet owner needs to meet to provide adequate welfare to their companion animal. There is no discrimination or age restriction to suggest that elderly

\footnotetext{
${ }^{11}$ Deborah Rook, 'For The Love Of Darcie: Recognising The Human-Companion Animal Relationship In Housing Law And Policy' (2018) 39 Liverpool Law Review 29, 37

${ }^{12}$ Animal Welfare Act 2006, s.14

${ }^{13}$ Animal Welfare Act 2006, s.9
} 
people will not satisfy these requirements in the same way those under the age of 65 would. It is likely that in care homes this would also be the duty of the care home staff under S.3 of the Act, as they will be potentially responsible for the animal on a temporary basis, depending on what level of care they provide to the animal. ${ }^{14}$ Many might question whether or not this would be their duty, but it is likely that they will fall under section this if there was a dispute.

Previous research conducted found that despite over $40 \%$ of care homes stating they accept companion animals, the reality is that in practice it is far less than that. ${ }^{15}$ We found that some care homes stated that they allowed pets but in reality they didn't. These discrepancies are proof as to why our research is so important, to ensure that elderly residents know their position when choosing care with a companion animal and whether or not pets are allowed in the homes they are choosing to live in.

\section{Benefits of the elderly owning pets}

The main focus of previous research into this issue is more about the benefits of companion animals on the elderly, as opposed to the practicalities and accessibility of the elderly living with their companion animal. There is a particular deficit in knowledge and research on this area of accessibility of care homes catering for pets and the hope is that our research will aim to fill some of these deficits.

A research report by CLH Healthcare in 2017 looked at the benefits of pet-friendly healthcare. ${ }^{16}$ The report stated that for a lot of older people (65 and above), their choice of pet would be a cat as they are less physically demanding as a dog, but are just as affectionate and sociable. They highlight that the big downside to pet ownership is the idea of having to give up pets when entering supported accommodation or care. In the report, Lori Palley says: "Pets hold a special place in many people's hearts and lives, and there is compelling evidence from clinical and laboratory studies that interacting with pets can be beneficial to the physical, social and emotional wellbeing of humans." 17

The report also suggests that pets can soothe and relieve anxiety in people with dementia. The Alzheimer's Society has discussed the positive impact that pets can have on dementia sufferers. ${ }^{18}$ It is suggested that any pet will benefit someone who suffers with dementia and will help to improve their quality of life. Pets bring a sense of unconditional love, friendship, and fun and, for people with dementia, it can help prevent feelings of anxiety or depression by reducing irritability, agitation, and loneliness. ${ }^{19}$

\footnotetext{
${ }^{14}$ Animal Welfare Act, s.3(1)

15 BlueCross, Care Home Pet Policies, <https://www.bluecross.org.uk/care-homes-pet-policies> accessed $30^{\text {th }}$ April 2019

16 CLH Healthcare, The Benefits of Pet-Friendly Care Homes, (7th August 2017) <https://www.clhgroup.co.uk/news-article/2017/08/07/the-benefits-of-pet-friendly-care-homes/254> accessed $30^{\text {th }}$ April 2019

17 Ibid, paragraph 7.

$18 \mathrm{lbid}$, paragraph 9

${ }^{19} \mathrm{Ibid}$. Please also see, Ian H. Stanley and others, 'Pet Ownership May Attenuate Loneliness Among Older Adult Primary Care Patients Who Live Alone' (2014) 18 Aging \& Mental Health
} 
Jane Fossey, clinical psychologist and vice-chair of Society for Companion Animal Studies (SCAS), states "A number of small-scale studies suggest that introducing animals into care homes can have positive effects for people with dementia. For example, spending time with visiting animals has shown to reduce blood pressure and anxiety, and improve social interaction and sleeping patterns. It can also reduce the late-afternoon restlessness that can affect people with dementia." 20

The obvious benefit of pets in care homes is also explained: the reduction in loneliness. The $\mathrm{CLH}$ Healthcare report discusses how seniors in care homes are likely to begin to feel cut off from the outside world, especially when mobility is an issue. A study by Stanley et al has shown that having a pet can help to prevent loneliness in seniors, cheer them up and even improve their quality of life. ${ }^{21}$ The study shows that pet owners were $36 \%$ less likely than nonpet owners to report loneliness. An interaction was found between pet ownership and living status, in which living alone and not owning a pet was associated with the greatest odds of reporting feelings of loneliness. ${ }^{22}$

Another obvious benefit of pets in care homes is the support they can provide to their owners. For elderly residents suffering from certain health conditions, such as hearing loss or vision loss, pets are an obvious and solid support system. Usually, assistance pets are dogs, but in some instances other animals can be used. ${ }^{23}$ Support dog uses now vary past just vision and hearing: dogs can now be trained to deal with other health conditions such as dog's ability to detect when a diabetics blood sugar is too low or too high, or their ability to sense panic attacks and calm anxiety. ${ }^{24}$

Here are just some benefits of having pet-friendly care homes as a whole, but our research sought to delve more deeply to the limitations and restrictions than the benefits. However, having the knowledge of the benefits that pets can bring to resident's helped shape the questions for the interviews.

An earlier study by the Joseph Rowntree Foundation in 2008, looked at 234 care homes and sheltered housing units in six UK cities replicated a study by the Joseph Rowntree Foundation in 1993 which highlighted the importance of pets to older people. Research psychologist June McNicholas, who conducted both studies, is disappointed that attitudes have changed little

\footnotetext{
${ }^{20}$ Ibid, paragraph 11

${ }^{21}$ Ian H. Stanley and others, 'Pet Ownership May Attenuate Loneliness Among Older Adult Primary Care Patients Who Live Alone' (2013) 18 Aging \& Mental Health

${ }^{22}$ Ian H. Stanley and others, 'Pet Ownership May Attenuate Loneliness Among Older Adult Primary Care Patients Who Live Alone' (2013) 18 Aging \& Mental Health

23 CLH Healthcare, The Benefits of Pet-Friendly Care Homes, (7th August 2017), paragraph 14 <https://www.clhgroup.co.uk/news-article/2017/08/07/the-benefits-of-pet-friendly-care-homes/254> accessed 30 $30^{\text {th }}$ April 2019

${ }^{24}$ Ibid, paragraph 15. Please also see Cindy Wilson, 'The Pet as An Anxiolytic Intervention' (1991) 179 The Journal of Nervous and Mental Disease; Mary M. DeSchriver and Carol Cutler Riddick, 'Effects of Watching Aquariums on Elders' Stress' (1990) 4 Anthrozoös; WP Anderson, CM Reid and GL Jennings, 'Pet Ownership and Risk Factors for Cardiovascular Disease in People' (1992) 69 Australian Veterinary Journal; Karen Allen, Barbara E. Shykoff and Joseph L. Izzo, 'Pet Ownership, But Not ACE Inhibitor Therapy, Blunts Home Blood Pressure Responses to Mental Stress' (2001) 38 Hypertension; Karen Allen, Jim Blascovich and Wendy B. Mendes, 'Cardiovascular Reactivity and The Presence of Pets, Friends, and Spouses: The Truth About Cats and Dogs' (2002) 64 Psychosomatic Medicine.
} 
in 15 years, despite growing evidence of the health and social benefits that pets bring. "We need to explode the myths that it is difficult, dangerous or time-consuming to have pets in care," she says. "A lot of fears from managers and staff are unfounded." 25

There is not much case law in this area, but one was found. Mrs $P$ (by her litigation friend, the Official Solicitor) v Rochdale Borough Council, NHS North, Central and South Manchester Clinical Commissioning Groups, ${ }^{26}$ concerned a financial Deputy's failure to consider bringing Mrs P's dog, Bobbie, with her into a care home after she suffered two strokes. Mrs P was devoted to her dog and it was the 'only living being with whom she shared any love. ${ }^{27}$ As the Deputy had not arranged for further contact with Bobby, amongst other wrongdoings, they were removed from being Mrs P's Depuyty. The judge stated:

"It may seem to those not well rehearsed in the needs of a person who owns a pet, in this case a person who no longer has capacity to make decisions about various matters, what the importance of a pet is in their life. The deputy only has to read any single reference in reports, assessments or statements of Mrs $P$ of how important Bobby is to her. Her Social Worker says in her witness statement to the court that:-

"I would recommend that of single most importance in her life is her dog and having some form of contact with her dog in the future if possible." 28

Whilst this case is not specifically regarding pets in care homes, it is a positive step that the court recognised the importance of the relationship between Mrs P and Bobby and the lack of this contact being unacceptable. It is hoped that cases in the future will also take positive steps for the elderly and their companion animals.

\section{Strains on other organisations}

A newspaper article by Holly Brockwell ${ }^{29}$ provides several other good points about why the elderly should be able to keep pets. One, the elderly are very unlikely to have big, messy and out of control pets and therefore aren't likely going to damage the property. Secondly, the number of animals that go in to shelters is high. An overall figure by the RSPCA alone found that they rescued and collected 102,900 animals during $2018 .{ }^{30}$ Another source said that in $2010,246,397$ cats and dogs entered the care of the participating organisations ${ }^{31}$. Of these numbers, 48,770 dogs were surrendered by owner and 45,899 cats were surrendered by owner (accounting for 94,669 of the animals in the study). ${ }^{32}$ It is hard to find a further break

\footnotetext{
${ }^{25}$ Blazina, C., Boyraz, G. and Shen-Miller, D., The Psychology of the Human-Animal Bond (Springer 2011)

${ }^{26}$ [2016] EWCOP B1

27 Ibid, paragraph 14

28 Ibid, paragraph 29

${ }^{29}$ Holly Brockwell, 'I Back Labour's Plan For Renters With Pets - Let's Stop Filling Shelters And Torturing Tenants' (inews.co.uk, 2018) <https://inews.co.uk/opinion/i-back-labours-plan-renters-pets-lets-stop-fillingshelters-torturing-tenants $/>$ accessed $28^{\text {th }}$ April 2019

${ }^{30}$ RSPCA, Facts and Figures < https://media.rspca.org.uk/media/facts $>$ accessed $30^{\text {th }}$ April 2019

31 Jenny Stavisky, Marnie L Brennan, Martin Downes, Rachel Dean, 'Demographics and Economic burden of unwanted cats and dogs in the UK: results of a 2010 census' (2012) 8(3) BMC Veterinary Research

32 Ibid, 7, table 2
} 
down of the number of those that were surrendered by elderly owners entering care, and these figures are not meant to represent this, but merely show the high numbers of animals entering shelters. Brockwell states that, "Landlords are the reason our animal shelters are overflowing. They don't mean to be, and they probably don't even realise, but they are." 33 This is not addressed in this research report, but is a focus for further research.

With this, the study found high numbers of "euthanasia" of animals was found. The study also suggested that other studies, such as Clark et $\mathrm{al}^{34}$, showed there was a significant increase even between 2009 and 2010 of ethuanised animal numbers. Anchor Trust published research in 1998, which highlighted that almost 40,000 pets are needlessly destroyed every year. ${ }^{35}$ This could be used to support an argument for promoting ownership of pets in care homes, as a method to help reduce the number of animals being euthanised.

\section{Other Jurisdictions}

Other jurisdictions have legislated on this issue and can be used as guidance and inspiration in England and Wales. The other jurisdiction focus on tenancy agreements and leases generally, rather than just focusing on care homes, but may be comparable and relevant in this area. In the Care Home Handbook ${ }^{36}$, Dr. Graham Mulley from Leeds University states that, some countries, such as France, Switzerland, the USA and Norway, have laws which oblige care home providers to accept companion animals belonging to elderly residents. In others it is left to the individual homes. Some accept no pets and others accept specific animals. Some refuse permanent pets, but will allow pets to be brought in by visitors.

\section{USA}

In the USA, Rebecca J Huss published a detailed article entitled 'No Pets Allowed: Housing Issues and Companion Animals' ${ }^{37}$, on the benefits of animals and the difficulties in remaining with them when living in rented accommodation. The article discusses, in depth, the benefits animals can have for their owners. Stating that pet owners report that they believe their pets relieve stress and are good for their health and the health of other human family members. ${ }^{38}$ She states that this belief is supported by 'increasing' numbers of research studies that have looked at the impact companion animals have on human health, and lists a number of studies. ${ }^{39}$ Generally, the research shows that contact with animals has a calming effect on people for example by reducing their blood pressure and cholesterol.

\footnotetext{
${ }^{33}$ Holly Brockwell, 'I Back Labour's Plan For Renters With Pets - Let's Stop Filling Shelters And Torturing Tenants' (inews.co.uk, 2018) <https://inews.co.uk/opinion/i-back-labours-plan-renters-pets-lets-stop-filling-shelterstorturing-tenants $/>$ accessed $28^{\text {th }}$ April 2019

${ }^{34}$ Clark CC, Gruffydd-Jones T, Murray JK. 'Number of cats and dogs in UK Welfare Organisations' (2012) 170(19) The Veterinary Record

${ }^{35}$ Anchor Trust, Losing a Friend to Find a Home: The dilemma of older people forced to decide between keeping their pets and finding a place to live (1998)

${ }^{36}$ Graham Mulley, Clive Bowman, Michal Boyd, Sarah Stowe, The Care Home Handbook (Wiley-Blackwell 2014) 38-39

${ }^{37}$ Rebecca J Huss, 'No Pets Allowed: Housing Issues and Companion Animals' (2005) 11 Animal L. 69

${ }^{38}$ Rebecca J Huss, 'No Pets Allowed: Housing Issues and Companion Animals' (2005) 11 Animal L. 69

${ }^{39}$ See generally, Cindy C. Wilson \& Dennis C. Turner Companion Animals in Human Health (Sage Publications 1998) discussing a variety of studies done on the impact of companion animals on human health; Delta Society,
} 
Huss points out that animals, under U.S. law are treated as personal property, ${ }^{40}$ as they are in the UK ${ }^{41}$ and other countries. In America, it is tradition that people have a legal right to own and control property and generally the common law will support the idea of "absolute" possession of property - although there are restrictions to the use of personal property. If we had longer to conduct our research, it would have been interesting to look in to the situation of, a person going in to a care home and not allowed to take their pet and whether you could argue they are being deprived of their personal property. ${ }^{42}$ This has the potential to be its own research in the future, as a way of promoting awareness for elderly pet owners' rights.

In America, landlords may restrict or prohibit the harbouring of an animal in a rental unit. Further, local laws may restrict the number of animals to be held on the property or impose other requirements relating to the ownership of the animal even if a person owns the real property where the animal is located. ${ }^{43} \mathrm{~A}$ restriction similar to some of the problems faced in the UK when it comes to renting properties.

Huss highlights the issues with the Elderly and Handicapped Housing. ${ }^{44}$ As part of the Housing and Urban-Rural Recovery Act 1983, Congress passed a rule entitled 'Pet Ownership in Assisted Rental Housing for the Elderly or Handicapped' (POEH). This rule provides that owners and managers of federally assisted rental housing for the elderly or handicapped cannot prohibit or prevent a tenant from owning common household pets. The rule does allow for some exceptions and the owner or manager of any federally assisted housing for the elderly or handicapped can remove any pet which is causing a nuisance or is a threat to the health and safety of other occupants or persons in the housing. ${ }^{45}$ This is a law that the UK does not have, but could definitely benefit from having or, at least, consider something similar to ensure that elderly residents have the right to keep their companion animals with them, even if there are some restrictions or limitations.

Since this rule was introduced, The Department of Housing and Urban Development (HUD) have established an extensive set of regulations to implement $\mathrm{POEH} .{ }^{46}$ The regulations provide that tenants are to be given notice of the rights they have under the law and are to be given access to any pet rules developed in accordance with the regulations. The pet rules themselves are divided in to discretionary and mandatory rules. ${ }^{47}$

Health Benefits of Animals Bibliography, (listing articles that report on studies finding companion animals affect human health in a positive way); Delta Society, Healthy Reasons to Have a Pet, (listing the results of studies that found various ways that companion animals improved the health of humans). Aaron H. Katcher, How Companion Animals Make Us Feel: Perceptions of animals in American Culture (Smithsonian Institution Press 1989)

${ }^{40}$ Gary L. Francione, Animals, Property, and the Law (Temple U. Press 1995); Orland A. Soave, 'Animals, the Law and Veterinary Medicine (4th edn, Rowman \& Littlefield 2000)

${ }^{41}$ Deborah Rook, 'For The Love Of Darcie: Recognising The Human-Companion Animal Relationship In Housing Law And Policy' (2018) 39 Liverpool Law Review 29

42 This is a human rights issue, specifically focusing on Aeticle 1, Protocol 1 of the European Convention on Human Rights. As it is a human rights issue, it will only apply to a public authority care home, not a private care home.

${ }^{43}$ See infra pt. IV (discussing local laws).

${ }^{44}$ Rebecca J Huss, 'No Pets Allowed: Housing Issues and Companion Animals' (2005) 11 Animal L. 69, 90-93

4512 U.S.C. s.1701r-l(b), (c).

${ }^{46} 24$ C.F.R. s.5.300 et seq. (2003).

$47 \mathrm{Ibid}$, at ss. 5.318, 5.350. 
The mandatory rules include that pet owners must have their pets inoculated in accordance with state and local laws ${ }^{48}$, sanitary standards must be set governing the disposal of pet waste, including specific limitations on the number of times a week that a pet owner is required to change the litter in a litter box ${ }^{49}$, pets are required to be restrained and under the control of a responsible individual while on the common $\operatorname{areas}^{50}$, and the pet owners must register their pets and update their registration at least annually. ${ }^{51}$ The discretionary pet rules provide some flexibility to reflect the needs of each housing project (supported accommodation). These rules allow reasonable limitations on the number of pets in each unit and specifically state that "project owners may limit the number of four-legged, warmblooded pets to one pet in each dwelling unit." ${ }^{52}$ Reasonable restrictions on the size, weight, and type of animals in the project may be mandated under the discretionary pet rules. ${ }^{53}$ Pet deposits are allowed; however, they are limited to the equivalent of one month's rent or to an amount set by HUD periodically, depending on the type of housing. ${ }^{54}$ There are also discretionary rules which set out the standards of pet care, for example, that pet owners may be required to control noise and odour caused by a pet ${ }^{55}$

If a tenant violates a pet rule, a procedure set out in the regulations sets out a minimum notice and meeting requirements before steps can be taken to remove a pet or terminate a pet owner's tenancy. ${ }^{56}$

The ideas presented in Rebecca J Huss' work provided some interesting points of thought and a host of potential reforms that could be made to the Law here in the UK. For example, the idea of regulations will provide protections for the owners and managers of the care homes and supported accommodation would be a positive change for the UK, as it will give them legal restrictions they can apply to the conditions of rent.

\section{Canada}

Under Sect. 14 of the Residential Tenancies Act 2006, 'no-pet' clauses are prohibited, but landlords are allowed to refuse to rent to someone with a pet, not including service animals. However, a 'no pet' clause in a lease is void, so signing a lease that has one doesn't give your landlord the right to evict you if you move an animal in, even if you previously agreed not to have a pet. ${ }^{57}$ The landlord can, however, apply to evict the tenant if the animal, for example, is causing the landlord or another tenant to suffer a serious allergic reaction, or they are interfering with the reasonable enjoyment of the property. ${ }^{58}$

\footnotetext{
$48 \mathrm{Ibid}$, at s.5.350(a). Discretionary standards allow pet rules to require pet owners to license their pets in accordance with State and local law. Id. at s. 5.318(f).

49 Ibid, at s. 5.350(b).

5024 C.F.R. at s. 5.350 (c).

51 Ibid, s. $5.350(d)$.

52 Ibid, s. 5.318(b)(1)(ii).

53 Ibid, s. 5.318(b)(1)(ii).

${ }^{54} \mathrm{Ibid}, \mathrm{s.} 5.318$ (c). At least one housing authority outside of the state of California has banned ferrets.

55 Ibid, s. 5.318(e) (2).

${ }^{56} 24$ C.F.R. S. 5.356.

57 Tribunals Ontario: Social Justice Division, Landlord and Tenant Board: Frequently Asked Questions, $<$ http://www.sjto.gov.on.ca/ltb/faqs/\#faq8> accessed 30 ${ }^{\text {th }}$ April 2019

${ }^{58}$ Residential Tenancies Act 2006, s.76 (Canada)
} 
Debbie Rook has argued that this can, "encourage dishonesty on the part of the tenant, or at least a failure to disclose the truth, as it is better for a tenant not to admit to having a companion animal until they have signed the lease." 59 Whilst this is a good comparable jurisdiction for introducing 'no pet' clauses, there are issues with it which need to be considered.

\section{France}

France was the first country to prohibit 'no pet' clauses in leases, the 1970 legislation ${ }^{60}$ declaring these clauses in residential tenancies are void. There is a requirement that the animal does not cause damage to property or disturbance to other occupants.

\section{Reasons against allowing pets in care homes}

\section{$\underline{\text { Age }}$}

Coren highlights that some people in Canada feel that the elderly shouldn't be allowed to adopt kittens or puppies, stating, "the board is divided on the issue of whether seniors should or should not be allowed to buy kittens, puppies, cats or dogs from them. One side says that the elderly should not be allowed to adopt pets from the shelter in view of the fact that if the human dies before them these pets will be orphaned. The other side suggests that this is age discrimination." 61

This is a very interesting view on elderly pet ownership and one that doesn't come up a lot in research. It is easy to agree the fact that elderly people could die when looking after an animal is just as likely as any other person. To make comments like this hold any weight, there would have to be some assessment of the elderly individual's health and condition, and sadly, the likelihood any condition or illness causing a soon-death. Coren agrees with this stating, "The argument that if the animal's owner dies sooner than their companion, the pet will be orphaned does not appear to me to have much merit. The same argument could be to suggest that single men or women in their $20 \mathrm{~s}$ and 30 s should not be allowed to have pets unless they have family living same city with them who would be willing to take over the pet should they die or become incapacitated". ${ }^{62}$ This is also, he argues, the same argument for those adults in the midst of their career and caring for a family, as they may find it difficult to care for a pet and, "the truth of the matter is that because of the bond that seniors tend to form with their pets, they will usually go out of their way to make sure that their pet is well looked after and there is someone who is willing to take care of the animal should they die before their animal companion".63

\footnotetext{
${ }^{59}$ Deborah Rook, 'For The Love Of Darcie: Recognising The Human-Companion Animal Relationship In Housing Law And Policy' (2018) 39 Liverpool Law Review 29, 39

${ }^{60}$ Article 10 and the Law of 9 July 1970

${ }^{61}$ Stanley Coren PhD, DSc, FRSC, Animal Shelters say "No puppies or kittens for the elderly" (January 2011) $<$ https://www.psychologytoday.com/us/blog/canine-corner/201101/animal-shelters-say-no-puppies-orkittens-the-elderly> accessed 30 ${ }^{\text {th }}$ April 2019

62 Ibid

$63 \mathrm{lbid}$
} 
This is another interesting point. The fact of the matter is, most elderly people need the companionship of their pets and often this companionship will provide them with a sense of purpose and routine - as mentioned previously. Given that an elderly resident has the capacity to care and look after the animal, it is fairly easy to argue that they will be able to provide a more constant care for the animals that a working man, woman or family would be able to. But again, this is all down to individual capacity and this would be something that would be necessary to monitor as a way of checking for any decline in health. He stated: "it seems to me that to deny seniors the companionship of a pet is simply an act of cruelty" 64 .

Based on a healthy elderly individual this is a statement that many would agree with. But it is all about the assessment of capacity and their ability to care for an animal, because if they cannot care for the animal, who will? This is something explored in our research.

\section{Health and Safety}

Another reason for not allowing pets is health and safety concerns. Animals could lead to lower levels of hygiene (especially hand hygiene), which could lead to issues regarding risk of common colds and illness. Another issue is the risk of infection or other injuries resulting from animals, for example, if there is a less friendly dog in a communal area that accidentally attacks someone. According to a publication by Ohio State University ${ }^{65}$, a recent study revealed gaps in protocols surrounding animals in long-term facilities, which contributed to higher risk of health and safety related issues.

The publication is more about having animals in the care home generally, as visitors or with residents as companion animals, but either way it makes interesting suggestions. The publication sets out ways to maximise benefits and reduce risk in facilities by taking simple steps:

$0 \quad$ 1) choose the best animal;

o 2) ensure safe interactions between people and animals;

0 3) require evidence of proper animal care; and

0 4) create animal safety and cleanliness protocols for your facility. ${ }^{66}$

These recommendations are very subjective and generic in nature. They would also be very hard to enforce, especially point 2 , as if anything was to happen that might carry some risk, and it would be too late to stop it. It is, however, just meant to provide a framework of the considerations a care home would need to make, and more detail would be needed to make an actual protocol for the facility.

They also recommend choosing good-natured animals to avoid bites and scratches - facilities should avoid animals that have a higher chance of making residents sick such as reptiles, amphibians, rodents and exotic species. ${ }^{67}$ For example, animals such as turtles, frogs and

\footnotetext{
$64 \mathrm{Ibid}$

65 Ohio State University, Animals in Ohio Long-term Care Facilities: Keep Residents Safe While Enjoying Pets (2016) <https://kb.osu.edu/bitstream/handle/1811/79017/Animals LTCF Brochure.pdf?sequence=9\&isAllowed=y > accessed $30^{\text {th }}$ April 2019

66 Ibid, 3

67 Ibid, 2
} 
other reptiles/amphibians commonly carry Salmonella (a bacteria), which can cause severe illness in some people, especially anyone who suffers with a weakened or otherwise compromised immune system (a condition that is common among the elderly population). Also rodents, farm animals and exotic animals can also frequently transmit diseases.

They suggest that the age of the animal could have an impact on health of residents. They state, "Keep residents safe by using only mature animals", suggesting that choosing animals over the age of 1 years will lower the amount of biting and scratching incidents as well as lowering risk of the animal carrying any disease. ${ }^{68}$ Ohio State have also issued guidance on ensuring safe interactions between residents and animals, and have published a draft protocol form for individuals to use to draft their own version. ${ }^{69}$

Despite this being a U.S. study, its advice could help the UK tackle the potential problems and concerns of allowing all care homes to accommodate resident's companion animals. The document makes some good recommendations on how to reduce the health and safety risks, especially the risk of disease and injury to both the elderly and the animals themselves, that are a cause for concern for many.

The Centre to Study Human-Animal Relationships and Environments also have similar recommendations, putting forward that care homes should provide plans of letting pets into care homes and, "To provide rational precautions for health and safety of people without unduly limiting the benefit of animals (as judged by the responsible administrator with consultation from a veterinarian, a physician and others)." 70 Further, residents, staff, and appropriate consultants should be in consultation in developing plans for allowing pets into care homes, consider the needs of some staff and residents not to be near the animals and consider the choice of the animal, including its purpose. Lastly, they advise that veterinarians should be advisors in selecting animals in a care home and "in developing a plan for promoting and monitoring the health and behaviour of resident animals as a continuing health maintenance program." 71

It is a fairly good attempt at a full-coverage list of recommendations for both visiting pets and residential pets. These recommendations are generally what you could expect (e.g. specifying the types of animals to be expected to be accepted in to the facility) as well as relevant points on the care of animals (vets providing leadership for monitoring health and behaviour etc) but there are also some gaps in the recommendations. More detail could be given on what sort of 'plan' could be in place and how this should be done. There should be more clarity regarding restrictions on where the animals are allowed and the minimum requirements of room size to house different animals, to ensure there is enough room for the animal to live comfortably. Moreover, there may be some difficulties for care homes to work with these

\footnotetext{
68 Ibid, 3

${ }^{69}$ Ohio State University, Model Animal Protocols for Long-Term Care Facilities, $<$ https://kb.osu.edu/bitstream/handle/1811/79017/Model Animal Protocols for LTCF.pdf?sequence=10\&isA llowed $=\mathrm{y}>$ accessed on $26^{\text {th }}$ April 2019.

70 CENSHARE, Research Abstracts on the Human-Animal Bond and Long Term Care <http://www.censhare.umn.edu/public-service-projects/companion-animals-in-care-environments/researchabstracts-on-the-human-animal-bond-and-long-term-care/> accessed on $20^{\text {th }}$ March 2019

${ }^{71}$ Ibid, 6
} 
recommendations, particularly financially affording a vet to advise them and monitor behaviour of the selected animals.

\section{Current moves toward pets in care homes}

A news article by the Telegraph, reported that "Elderly people should be allowed to keep their pets when they move into a care home, preventing the needless destruction of thousands of animals every year." 72 This has been put forward by Nigel Waterson, and "his Care Homes and Sheltered Accommodation (Domestic Pets) Bill would ensure many elderly people would be able to keep their beloved animals when they have to leave their own properties." ${ }^{73}$ This Bill's aim was to create a legal presumption that pets could accompany the elderly into care homes and sheltered accommodation, unless an exception can be justified, such as the safety of the other residents.

This Bill, though reaching its second reading in March 2010, ultimately failed, due to a general election. There was support for the Bill across parties and if a similar Bill is introduced in the future it may stand a chance. There have been moves towards dealing with 'no pet' clauses, however, discussed below.

\section{Scottish Labour Party proposals}

The Scottish Labour Party, in April 2018, launched their 'paws clause' campaign, which would guarantee that owners can keep their pets if they move in to supported accommodation or care homes and also extends to those seeking temporary accommodation for the homeless. ${ }^{74}$

This movement by Scottish Labour shows that we as a nation really do care about our furry friends and want to protect them and their owners' rights. The inclusion of care home in this campaign is positive, as many proposals for change are focused on rental accommodation only, and it is refreshing to see political parties acknowledge the need for change for the elderly too.

The Scottish Labour Party are in consultation with landlords, tenants, care homes and temporary accommodation providers, to assess this possibility. ${ }^{75}$ Despite agreeing that the default should be for pet owners to stay with their pets, it is important to see how plans like this will frustrate landlords and cause them to lose rights and control over the property as landlords.

\footnotetext{
${ }^{72}$ Elderly people 'should be allowed to keep pets in care homes' The Telegraph (5 ${ }^{\text {th }}$ March 2010) <https://www.telegraph.co.uk/news/uknews/7377220/Elderly-people-should-be-allowed-to-keep-pets-incare-homes.html> accessed 30 $0^{\text {th }}$ April 2019 


\section{West Berkshire Local Government Policy}

West Berkshire local government have application forms that can be filled out in order to potentially take pets with you in to care homes. ${ }^{76}$ It does state, however:

"4.4. There is no guarantee that the care home can continue to care for a service user's pet in the event that the pet outlives their owner. The arrangements for managing this circumstance must be discussed and agreed with the service user / family / representatives as part of the admissions process." ${ }^{77}$

They also issued 'Guidelines for the care of a pet in a care home' ${ }^{78}$, however, the guidelines have not been updated or extended since 2014, which is now 5 years ago. Some might say that it may be outdated with the needs of society today, or maybe it has worked effectively so has not needed to be altered in the time, but there does not seem to have been any further work on this.

\section{BlueCross}

The BlueCross have a section on their website how the public can contact their local MP to help ensure all care homes have a pet policy. The website doesn't go in to detail about what this is and how effective it is but there is a template letter for people to use to send to their local MP. There is also a link for people to use to find their local MP too. ${ }^{79}$

76 West Berkshire Council, Procedures for bringing pets into care homes (May 2014) $<$ http://www.westberks.gov.uk/CHttpHandler.ashx?id=4637>_accessed 30 ${ }^{\text {th }}$ April 2019

77 Ibid, paragraph 4.4

$78 \mathrm{Ibid}$

79 BlueCross, 'Care Home Pet Policies: Ask your MP to help ensure all care homes have a pet policy' $<$ https://www.bluecross.org.uk/care-homes-pet-policies> accessed $26^{\text {th }}$ April 2019 


\section{Research Methodology}

\section{Ethics}

Ethics was granted by Northumbria University's Ethics Committee. We did have some issues surrounding ethics, which is discussed in more detail in the limitation section below. All participants were sent a consent form, which contained information about the study and how the data was managed, ${ }^{80}$ prior to the interview taking place. This allowed the participants time to ask the research team any questions they may have had about the study prior to it taking place.

\section{Sample}

To recruit participants, a recruitment email/letter was sent around care homes in Newcastle upon Tyne and North East area. ${ }^{81} \mathrm{~A}$ list was created of 23 care homes in the area with their names, address, phone, email and their pet policy. All 23 potential participants were contacted either through email or letter. Because there were no responses from the first correspondence after a month, follow-up calls were made. After the follow-up calls were made, 5 potential participants agreed to take part in the research, which was then narrowed down to 4 participants, as one fell out of contact. However, after one of the interviews, we got an interest from a care home manager outside of our initial recruitment area. Ultimately, 5 participants were recruited to participate in the research.

Interviews were scheduled using phone calls and follow up by email. Both telephone and faceto-face interviews were used for this sample. There were 3 face-to-face interviews and 2 telephone interviews, and the participant had the option to choose the most convenient one for them. For the face-to-face interviews, we travelled to the care home for their convenience and, similarly, if they opted for a telephone interview, we called them. For the face-to-face interviews a consent form was signed, whilst for the telephone interview the consent was gained orally, recorded using a Dictaphone so later it can be transcribed for evidence of consent.

\section{$\underline{\text { Interviews }}$}

We have used a qualitative research method to obtain empirical data for this research project. This focuses on describing the meaning and central themes of the research and was useful to enable us to get to the experiences of the care homes managers, which ensured the depth and the breadth of the research.

To obtain this type of data, we conducted semi-structured interviews, which provided us with some good and practical data. It allowed us to plan specific questions, whilst also allowing room for any clarifications and explorations of interesting topics and comments. This keeps the interview under control, but are easily adapted when needed, whilst keeping the interviews similar.

\footnotetext{
80 Please see Appendix 1 for the consent forms.

${ }^{81}$ Please see Appendix 2 for the recruitment emails.
} 


\section{Questions}

Our interview questions ${ }^{82}$ were divided into four parts, focusing on:

1) Whether pets were allowed in the care home.

2) The second part of questions were asked if the participant answered 'yes' to the first question. These questions established the background of why they allow pets in their care home, the benefits they have experienced, and how they manage this.

3) The third set of questions were asked if the participant answered 'no' to the first question. This set of questions explored why pets are not allowed in their care home, the company policy around this, and whether they allow animals in to visit.

4) The fourth set of questions looks at the legal aspects. The questions that are included are whether they know about any laws surrounding pets in supported accommodation and their thoughts around potential legislation banning 'not pet' clauses.

To make the interview process easier to follow, a flow chart was created showing the links between the questions. ${ }^{83}$

\section{Analysis}

To analyse the data collected from the interviews, thematic analysis was used, as we felt it the most appropriate for this research project. It emphasises pinpointing, examining, and recording patterns themes within data. ${ }^{84} \mathrm{~A}$ theme captures the important data in relation to the research question and represents some patterned meaning. ${ }^{85}$ Thematic Analysis consists of 6 phases:

\footnotetext{
82 Please see Appendix 3 for the interview plan.

83 Please see Appendix 4 for the flow chart.

${ }^{84}$ Virginia Braun and Victoria Clarke, 'Using Thematic Analysis In Psychology' (2006) 3 Qualitative Research in Psychology.

85 Ibid, 82
} 


\begin{tabular}{ll}
\hline Phase & Description of the process \\
\hline 1. Familiarising yourself with your data: & $\begin{array}{l}\text { Transcribing data (if necessary), reading and re- } \\
\text { reading the data, noting down initial ideas. }\end{array}$ \\
\hline 2. Generating initial codes: & $\begin{array}{l}\text { Coding interesting features of the data in a } \\
\text { systematic fashion across the entire data set, } \\
\text { collating data relevant to each code. }\end{array}$ \\
\hline 3. Searching for themes: & $\begin{array}{l}\text { Collating codes into potential themes, gathering all } \\
\text { data relevant to each potential theme. }\end{array}$ \\
\hline 4. Reviewing themes: & $\begin{array}{l}\text { Checking in the themes work in relation to the coded } \\
\text { extracts (Level 1) and the entire data set (Level 2), } \\
\text { generating a thematic 'map' of the analysis. }\end{array}$ \\
\hline 5. Defining and naming themes: & $\begin{array}{l}\text { Ongoing analysis to refine the specifics of each } \\
\text { theme, and the overall story the analysis tells; } \\
\text { generating clear definitions and names for each } \\
\text { theme. }\end{array}$ \\
\hline 6. Producing the report: & $\begin{array}{l}\text { The final opportunity for analysis. Selection of vivid, } \\
\text { compelling extract examples, final analysis of }\end{array}$ \\
selected extracts, relating back of the analysis to the \\
research question and literature, producing a \\
scholarly report of the analysis.
\end{tabular}

Diagram 1 - The Stages of Thematic Analysis 86

The first phase involved us familiarising ourselves with the data. All of the researchers transcribed interviews and checked them back against the recording to ensure accuracy. Transcribing is a good way to familiarise with the data as it requires you to not only listen to the interview again, but also engage with it and comprehend everything said. ${ }^{87}$ All of the researchers transcribed an interview, which was checked back against the recording.

Phase 2 involves generating initial codes, which are an identifying feature of the data and appear interesting to the researchers. ${ }^{88}$ We each took a copy of each of the five transcripts and began coding them. We approached the coding in different ways. Rachel used the computer system NVivo, whereas the rest of our group opted to simply use pen and paper. We did this separately to increase reliability of our coding and to ensure that we were each finding each of the codes, rather than deciding together what they were.

The purpose of coding is to use numerous code words to display what each statement in the interview is saying and to begin to see themes emerging. In terms of creating themes, we sat together and wrote down all the codes on a whiteboard and then had a lengthy discussion of recurring codes and how the codes could fit together in to themes. This was reviewed by the research team and transferred to diagrams, working through phases 3 - 5 .

\footnotetext{
${ }^{86}$ Ibid, 87

${ }^{87}$ Catherine Kohler Riessman, Qualitative research methods: Vol. 30 Narrative Analysis (Sage Publications 1993)

88 Virginia Braun and Victoria Clarke, 'Using Thematic Analysis In Psychology' (2006) 3 Qualitative Research in Psychology, 88
} 
We came up with 6 themes:

1. Responsibility (Care of animals and Care of Residents)

2. Benefits

3. Costs

4. Choice

5. Policy

6. Law.

We incorporated all the codes in to one or more of these themes and we will use these themes to guide our discussion below.

\section{$\underline{\text { Limitations }}$}

There were some limitations that we had experienced during this project. One of them was that the interviews with care home residents could not be conducted as originally planned. This was due to the potential lack of capacity to consent, which raised some ethical issues, and this meant that we were unable to conduct interviews with elderly residents. For example, some of the potential participants might have had to require the consent from a family member or the NHS. It was not feasible in the timeline that we have been given to acquire the consent from all the parties and we made the decision not to conduct the interviews with the elderly residents. However, we believe that this decision will not impact the aims and objectives of the research project, as the main objectives were to gain insight from the care home managers rather than those of residents. Further, the managers are in the best position to explore why pets are, or are not, allowed in their care homes and the issues surrounding this. The outcomes of the research project were not affected due to this ethical issue.

Another limitation experienced in this research was a lack of contact and delay in getting in contact with participants. Some care home managers needed persuasion to take part in the research and reassured of the confidentiality. Further, for some care home managers, it was difficult to take time out of their duties to speak with us. There were instances where they were not available for some time or where interviews had to be rearranged due to unforeseen circumstances. Even though this delayed the interview stage to some extent, it did not have a substantial impact on the project.

The small sample size can be seen as a problem by some. ${ }^{89}$ Ideally, we would have liked a sample size of 20-25 participants. The number that would have been better would have been between 20-25 care home managers. However, for a pilot study, 5 participants have given us a good idea of what is happening in this area and what changes could be recommended. We appreciate that the findings cannot be generalised, and that other areas of England may find different results, but we are not making claims of application to other care homes and we

\footnotetext{
${ }^{89}$ For example, please see Bryan Marshall, Peter Cardon, Amit Poddar and Renee Fontenot, 'Does Sample Size Matter in Qualitative Research?: A Review of the Qualitative Interviews in IS Research' (2013) Journal of Computer Information Systems 11
} 
acknowledge that further research is needed. ${ }^{90}$ This has not affected the research as through interviewing 5 different care homes allowed us to gain insight into all perspectives of pet ownership when moving into a care home/supported accommodation.

${ }^{90}$ This kind of research was appropriate for the Student Law Office at Northumbria University. If others would like to take this design and conduct the study in other areas of England, this is welcomed, to create a larger study. 


\section{Results and Discussion}

As was found through the research, the definition of "pet-friendly" has been widely interpreted. ${ }^{91}$ All of the managers involved in the study claimed to have a pet-friendly care home. However, the levels differed quite remarkably. All of the participants believed that the presence of animals was quite beneficial to some residents and that this should be facilitated to ensure residents' choices and preferences were respected.

All of the managers said that they do allow animals into the care home in some way. However, the definition of allowing pets differed. Three out five allowed pets to live in the home, only after assessing the situation in the house, the needs of the elderly owner and the type of animal, but had no pets in residence at the time of the interviews. The greater the risks are, the less likely it would be to allow pets in. Two out of five, only allowed visiting animals.

A number of concerns were repeatedly mentioned both about the live-in pets and also about the visitor pets:

- Is it practical/feasible to allow pets in?

- What kind of animal it is and level of care it needs?

- Is the owner capable of looking after them and, if not, who would look after them?

- Who would clean, walk and feed the animal?

- Who would take them out to do their business?

- Would the home have the right size and facilities to fulfil the pet's needs?

- How many animals can the home accept overall?

- What if the other residents do not like pets?

Other factors to take into consideration included:

- The wellbeing of other residents

- Some of the residents do not like to be around animals, sometimes due to medical reasons, e.g. asthma

- The issues with hygiene and infection control (i.e. cats litter)

- Safety issues (i.e. residents might trip over an animal)

- Noise levels

91 The Cinnamon Trust, 'Pet Friendly Care Home and Retirement Housing Register' <https://cinnamon.org.uk/pet-friendly-care-homes/> accessed $8^{\text {th }}$ January 2019. 


\section{Benefits}

\section{Graph displaying the type of benefits recognised}

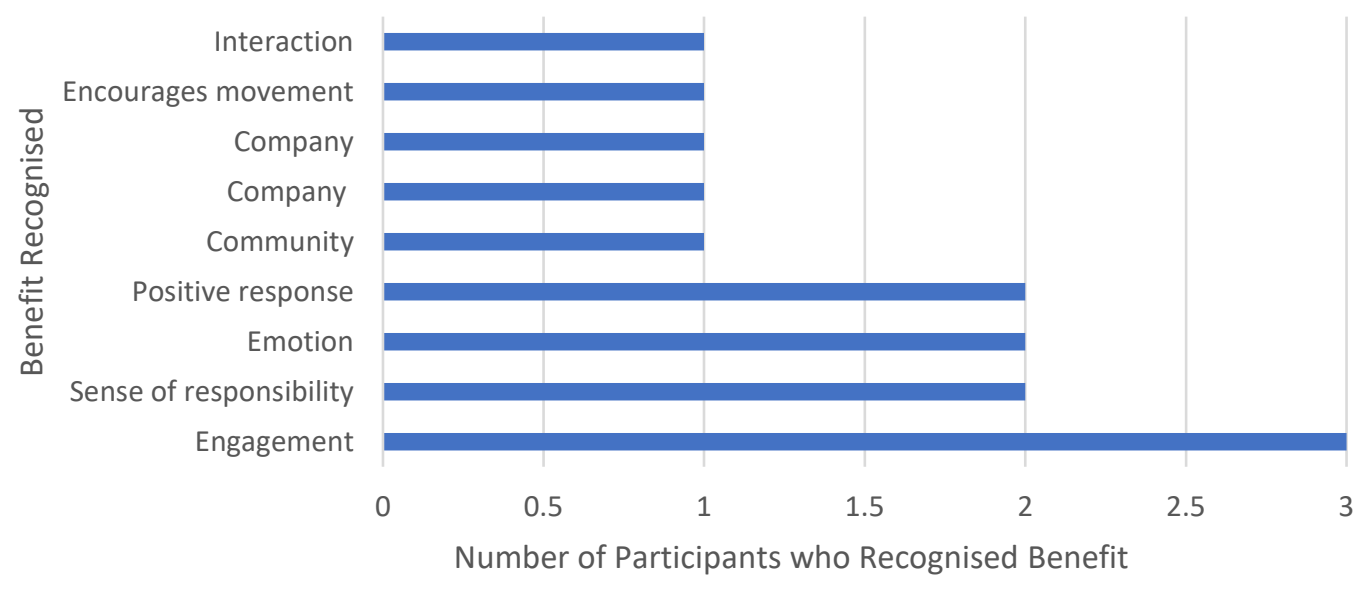

Graph 1 -Displaying the kind of benefit of pets in care homes recognised by the participants

All the participants have recognised some benefits of having pets around the elderly residents. The types of benefit recognised are mental health, physical health, positive response, emotion, interaction, encourage movement, company, community, engagement, and sense of responsibility, as shown in Graph 1. The horizontal axis shows the number of participants that have recognised the specific benefit. The most common response was elderly engagement with the pets. Three out of five participants felt that the residents were more engaged when an animal is around, both physically and mentally. The background research also confirms this recognising older adult owning a pet were more likely to engage in physical activity than non-dog or non-pet owners and previous research highlights that dog owners engaged in greater overall physical activity. ${ }^{92}$ This demonstrates that pets do have an impact on the elderly resident through the physical and mental changes can be seen when an animal is around the residents.

The next most common benefit recognised by managers is the mental health benefits on the elderly, which includes the sense of responsibility, positive response and emotion. One participant has stated:

"Most of our dementia residents are on top floor, they get a lot of satisfaction from the ponies coming up there. They are really gentle and you just see the look on their faces and respond to them. You know some of our ladies, really show a lot of interest in things but when they come in, you can see their faces and eyes light up. Like when they see a baby. It is quite nice."

Another one has said:

92 Roland J. Thorpe and others, 'Physical Activity And Pet Ownership In Year 3 Of The Health ABC Study' (2006) 14 Journal of Aging and Physical Activity 
"Most people would have had pets most of their life, so trying to keep everything that they've always had, it's a big importance, it's sense of self and responsibility. Just to taking the dog out for a walk, it benefits, not only the mental state, but also the physical state. It does get people up and mobile, having a dog, moving about."

It is clear that pets bring positive mental benefits to the residents. Most of the participants have noted that there is some element of enjoyment perceived by the resident whether it is an expression of positive emotion or having a cheerful reaction to animals. This is affirmed by several research reports, that by having an animal around it can bring a sense of unconditional love, friendship, and fun and, for people with dementia, it can help prevent feelings of anxiety or depression by reducing irritable, agitation, and loneliness. ${ }^{93}$ This has also been acknowledged in the Court of Protection in the case of Mrs $P$ (by her litigation friend, the Official Solicitor) $v$ Rochdale Borough Council, NHS North, Central and South Manchester Clinical Commissioning Groups, ${ }^{94}$ highlighting the importance of the elderly having their pets with them and that without her dog her quality of life is affected. One participant stated:

"I remember one, a dog that came with the resident, the dog was very elderly as well as the resident, and that's why because they both been together so long that it just would have had a complete negative impact to separate the two..."

Thus, some care homes do appreciate the need for an elderly person to remain living with their companion animals and that separating them is worse for that resident. This is another instance of it being decided on a case-by-case basis.

The less commonly recognised benefits are social benefits, such as community, company, and interaction. This could be due to a lack of information available about the social benefits that pets provide, as it can be seen that most research surround the mental and physical benefits rather than social benefits that the pets offer. The previous researches focus on the elderly person quality of life and combatting loneliness, ${ }^{95}$ which was discussed by a participant when asked if they had done any research around the benefits of pets for the elderly:

"As you know it's quite widely appreciated that pets do offer such positive benefits. Erm... especially with the regard to elderly people and combatting loneliness. It is just general information that we have picked up."

These benefits that are recognised have impacted on the manager's decision to have visiting pets, which can be either relative bringing them in or having a pet therapy. All the care home managers that have been interviewed have said that they allow a family member to bring their pet to the care home. One of the participants has stated that when a pet is brought in,

93 For example, please see CLH Healthcare, The Benefits of Pet-Friendly Care Homes, (7th August 2017) $<$ https://www.clhgroup.co.uk/news-article/2017/08/07/the-benefits-of-pet-friendly-care-homes/254> accessed $30^{\text {th }}$ April 2019

94 [2016] EWCOP B1

95 Ian H. Stanley and others, 'Pet Ownership May Attenuate Loneliness Among Older Adult Primary Care Patients Who Live Alone' (2014) 18 Aging \& Mental Health 
they don't let them have contact with any other resident and they go straight to the resident's room due to the health and safety of other residents and staff. Other care homes have a more communal feeling when a pet comes to visit, the manager allows the pet to be in a communal area so the residents that wish to see the animal have a chance to, which benefits all of the willing residents. In this case, the manager also weighs in the fact of those who do not like animals and allocated the space available based on that, so all the residents can feel comfortable in the care home. The visiting pets allow the residents to enjoy the benefits of the pets without assuming too much responsibility for them. One participant had pointed out:

"we have pets regularly, with different relatives who come in...... majority who live in here have got one form of dementia or another and all these mental health issues. Many of the people are not mobile and are not able to take the dogs out for a walk."

The pet therapies are either from a charity or private pet hires. One of the most popular in Newcastle upon Tyne was the pony hire. Three out of five participants have stated that they hire a pony to walk around the care home and visit residents in their rooms. This service is used quite frequently as one of the participants had stated that it is getting "more and more difficult" to book it and that they have gone from getting them every couple of years to now booking the visit once every couple of months. There are other pet therapies used, such as the charity Pets as Therapy (PAT), private hires including ponies and reptiles, as well as people bringing in dogs. These are all very popular ways for the elderly to enjoy animal companionship. This has been confirmed by various charities including Blue Cross and the Cinnamon Trust. ${ }^{96}$

\section{$\underline{\text { Costs }}$}

When discussing the cost of having a pet in the care home, it had included the cost of food, damage to the property, vet, staff costs, and potentially extra pay. All care home managers have stated that the cost of the food is the resident's responsibility which would also include other necessary supplies to look after the pet. This is understandable because the resident would need to pay for these supplies if they did not live in a care home.

\section{Veterinary care}

Another cost that would have to be considered by the elderly resident is the cost of the vet. No care home that participated in the research has on-site vet, which is understandable as those homes which allowed pets did not have many to justify getting a vet on the premises. However, the care homes are willing to assist the elderly in getting the pet to and from the vet if this is needed, for example, a family member can't take them. Others wanted the continuation of a specific veterinary practice to, "try and maintain that normality for them."

\footnotetext{
96 'The Cinnamon Trust - The National Charity For The Elderly, The Terminally III And Their Pets $<$ http://www.cinnamon.org.uk/home.php> accessed 9 December 2018.;

'Pets As Therapy: Animals Helping Humans' <https://petsastherapy.org/> accessed $26^{\text {th }}$ November 2018;

BlueCross, 'Care Home Pet Policies: Ask your MP to help ensure all care homes have a pet policy' <https://www.bluecross.org.uk/care-homes-pet-policies> accessed $26^{\text {th }}$ April 2019
} 


\section{Staff costs}

Some care home managers have stated that they are there to assist whenever possible rather than completely take care of the animals. This ties in with the staff costs of those who allow pets, as they do not charge extra for helping to care for it. One of the participants pointed out that their view of a pet living in a care home is an "extension of the resident" and animal care is part of the needs of the elderly. However, those participants that have enforced no pet policies have an opposite view on the staffing of caring for the animals. When asked if they would consider ever allowing pets one stated that the cost of any extra care/help needed would not be covered by the care home and "the staff are here to look after the person, not their animals." This included any help with the care of the pet and the resident would have to pay staff for the time spent on the animal. It is understandable that this would be a situation in big care homes where there are lots of elderly residents that all need constant care. Taking time to care for an animal could seriously impact on the general duties of the staff which would make the extra pay reasonable. One participant stated, "We can't accommodate every couple of hours having one of the staff taking a dog for a walk, but I can accommodate 15 minutes every couple of days for a cage to be cleaned out." Further, one mentioned it would be the same costs as the care for the person:

"So for example if we had to go for prearranged appointment to hospital or health centre for an appointment, if they are accompanied by member of staff, then the charge is 114 per hour for that. They would be talking away the job that they are supposed to do. You are looking at $f 14$ as minimum per hour for this. It is a lot of money."

This may justify their position on having enforced a no pet policy, as the cost of owning a pet in a care home can be substantial.

\section{Damage}

The damage that can be caused by pets, and the cost of that damage, is another deterrent, causing some care homes to impose a no pet policy. Those homes that do not allow pets have recognised that animals can cause a lot of damage, including urinating inside, chewing and noise levels. However, some care home managers interviewed saw the damage as being not a big problem and stated "There might be damage, but it's something that we can always rectify as and when we need to." They have stated that carpets can be easily replaced as even the elderly could have accidents on them: "we do replace carpets quite regularly, they would be damaged by the residents themselves". A participant had stated that if there was any damage to the fire door "it would be at the expense of the person who's in the room". 


\section{Responsibility}

Responsibility was one of the main concerns of the participants in the study. All of the participants mentioned that, responsibility is something that they must look into properly before letting a pet in. The main question that came up repeatedly was "who would be responsible for looking after the animals? This was covered above in relation to costs and staffing.

As stated above, one of the participants said they classify the pet "as part of the resident's needs" and an animal is an extension of the resident and "in order to promote the wellbeing of the resident, you need to take care of the animal." The staff at a home that does allow pets will help look after a pet if the resident deteriorates in health. However, as the amount of time staff can spend to look after an animal is not unlimited, again the care for the residents always take priority.

\section{Visiting Animals}

As stated above, all of the homes allowed pets to visit, regardless of whether they allowed to live on the premises. In fact, one participant mentioned that animals coming to visit "is the best next thing". They all agreed that relatives looking after the animal and bringing it to visit a couple of times a week would be ideal, as that would reduce the staff work and would also significantly minimise the risk of accidents and the threat to health and safety. The people who have had a history of having pets usually welcome the idea of pets being around. These animals included, dogs, ponies, reptiles, etc. The elderly who have lost their pets also - one case was mentioned - can see animals visiting which would cheer them up.

\section{Residents Care}

Residents' care always took priority over animals. One of the managers said, in their home, residents are encouraged to be independent, but the staff would help the residents in any way possible to look after the animals, such as cleaning them, exercising animals, feeding them, etc. However, it was said that they can only accept a small number of pets in that case, as more would impact on the staff time.

If the other residents had a safety issue, or allergy issue with the animal, or they just simply did not like animals, the home would be reluctant to accept any pets. There was a couple of mentions of some residents having had to give their pets to family, because the animals were a risk or a nuisance to other residents. Allowing pets in seems like it is on a case-by-case basis, and is decided upon residents needs within the home at the given time.

\section{Dependency and Deterioration}

The three managers that allow pets to live in the home, spoke about the benefits of having pets for the elderly. One talked about how in their home they encourage the residents to be active and independent and having pets to look after would give them that sense of purpose. However, the majority said that if the elderly loses her/his ability to care for the animal and becomes unable to care for their pet, then they would refuse to accept the animal. 
Almost all of the managers of the homes allowing pets said that, if the resident feels well enough to look after their pet, they are more than happy to support the resident to care for the animal, as the animal is a part of the owner's life and taking the benefits into account, the home would gladly accommodate the animals as well.

There was mixed views as to what would happen if the health of the elderly person were to decline. One participant stated they would try to accommodate for this:

"We would try and keep the pet as long as it was possible. Sometimes families will come in and they will do care for the pet, take it out. If that was unable, we would try and accommodate as much as we can, 'cause sometimes people decline only short term and they will get back on their feet. But if it somebody that's been around for a long time with the person, and they have a lot of love for that animal and we will try and keep here, as long as possible."

This was a very accommodating and pet friendly care home, so it is not surprising that they would like to keep the pet with its owner for as long as possible. They even said that, "some of the staff would take the dog on, and bring it in on certain days when they were on shift, so that the person still got to see the dogs." There were other care homes who agreed that they would help to look after a pet when the elderly person's health declined and that staff had taken the pet on themselves.

If the deterioration of health in the resident is permanent, however, then they encourage the relatives to take on the pet, and bring them to visit. This was often a decision made as the elderly person was coming into the care home. For example, one participant stated that there:

"was a lady who had a springer spaniel, some sort of spaniel, and we didn't let the dog come...so her daughter had the dog and she would bring it in regularly, so it was kind of the next best thing so. But to be honest, she wouldn't have been able to look after it herself. So it would have been something the staff would have had keep on top of..."

It really did depend on the size of the care home and how many members of staff they had to how accommodating they could be in this situation.

Hygiene, Health and Safety

Most care homes told us that they had residents with some form of dementia and that some of them are at the high dependency. Some residents lack capacity, they lack insight and awareness and are usually totally dependent on staff. Some have a weak eyesight and some suffer from breathing issues, such as asthma. 
In assessing which animals can come and live in the home, and which residents can have their pets with them, they weigh up the positives and also the risks threatening the health and safety of the resident owner, or other residents. If the animal is a cat, the cat tray might cause hygiene concerns and perhaps other health issues. The cat fur might cause breathing problems for asthmatic residents.

It was also suggested that, if a resident has an eyesight problem, it is quite likely that an animal could cause the resident to trip over and fall. Another concern that was talked about was the presence of wheelchairs, walking frames, buzzers going off all the time, and the animals' reaction to those. For example,

"we recently have been asked to assess a resident who had their own dog but because of the residents that we have on that floor at the moment use walking frames. We had to outweigh the risk for the residents on that floor which is sad because to see that joy that pets bring when they visit and things like that."

If the animals are not used to being around other people and the presence of such equipment would have a negative effect on them, then the animals must leave, as the risks are greater than the benefits in that instant.

Some residents do not like animals of certain type and some do not like to be around animals at all. There were quite a few instances where participants spoke about other residents being scared of animals, for example:

They have been quite recently. If it would have been on that floor a month ago, that would have been a possibility but again residents have changed on that floor, and their individual needs that I'm lead by and at the moment the lady on that floor is really scared of animals. It is their fear. Also, I have a lot of people who have high risks such as walkers and rollators.

Others have talked about not wanting to "isolate anyone in their own home" by allowing an animal to live in the care home which they are scared of. Some stated this is why visiting animals were better, because they could make sure that resident was in a different room when the animal was on the premises. One participant will move a visiting dog to a different room, if a resident who does not like dogs wants to be in that room. It seems that all care homes will cater for the resident and take their needs into account when animals are in the home. However, one of the managers said that they accept pets, because their presence makes the home feel "more homely".

\section{Accidents and Incidents}

There were minimal incidents, we were told of, involving animals in the care homes. One incident mentioned by one of the participant involved a dog jumping on a resident and they suffered from a skin tear on their knee. That dog was not allowed on the premises again. Another participant talked about a cat scratching walls and bringing dead birds into the home, which apparently the staff weren't very happy about. However, there were no other incidents 
mentioned by any participants of animals causing accidents. Some said that they hoped the "benefits outweigh the potential for risk" and this is why they allowed animals to visit.

\section{Policy}

Another theme discovered in our research was policy and this was a significant factor when making the decision to either allow pets in the home or not. As stated above, two of the care homes did not allow pets and they stated this was due to their policy. Many of the care homes visited had their own written pet policies with their own guidance on if and under what circumstances pets may live in the home with their owners.

Generally, these pet policies came from an aspect of the wider company policy for all their homes, some of which we managed to obtain copies of from two participants. The first one came from a care home that did not allow pets. The policy states that they do, if it is assessed that the animal is suitable, such as breed and size, how capable the elderly is to look after the pet and that the room is suitable for the animal. Even though this policy is in place, the manager did not allow any pets, mainly out of concern of who will look after the animal. The second pet policy came from a care home who does allow pets to live in the home. This pet policy provided pets are allowed, considering the kind of animal, the ability of the resident to care for the pet, where the pet can be kept and whether it will cause a nuisance or risk to other residents. It states that care staff will not help look after the pet, but in practice this was happening and the staff seemed to be very involved. It also provided guidance as to the responsibilities of the staff in terms of hygiene and infection control. This contrasts the first policy, which states the responsibilities of the residents and requirements of having a pet, but has nothing for the management. Both policies also state that they will help the elderly person re-home their pet, by putting them in contact with relevant organisations.

Therefore, it is clear that there is much influence coming from the managers of care homes, as to whether pets are allowed, rather than the actual company who owns the care home. There may be policies in place, but they are not strictly followed.

Having said this, we had evidence from one care home that hinted at some managerial influence. They were asked if their pet policy was manager dependent, to which they responded by saying "There is that element and the decision would be definitely influenced by the manager". This highlights that there is evidence of having some choice across our limited sample and that managers can be influential as to whether a home is pet friendly or not. This is consistent with the situation discussed in Rook's article, of Darcie being removed from a care home and his owner seeking alternative accommodation, due to a change of manager and their resistance of companion animals in care homes. ${ }^{97}$

Another manager had a particular interest in providing democracy for their residents. The home had a strong portfolio of situations they put to a vote for all residents in order to find out their views on different proposed idea to allow the people to have their say. One example

\footnotetext{
97 Deborah Rook, 'For The Love Of Darcie: Recognising The Human-Companion Animal Relationship In Housing Law And Policy' (2018) 39 Liverpool Law Review 29
} 
was given of a resident suggesting that they should raise a flag if a resident died, this wasn't agreed to by a majority and therefore didn't happen. They stated that if they were to allow pets, they would do the same thing if the issue of in-house pets were suggested by the management or residents alike, and:

"Erm, I think...you know, hopefully, I mean we have residents meeting here and hopefully we run the house to suit the needs of the residents, like a democracy".

This is an idea that could work across many care homes, to make the decision about what the residents' want as much as what the animals and pet owners want.

\section{Home Layout}

Another concern raised on numerous occasions was the issue of whether or not the home layout was practical for animals or whether it "particularly lends itself very well" to them living there. One home stated that they allow pets on a certain floor so that they are away from those who do not like animals. This is a good idea and a good compromise to allow a home to have animals without infringing on others in the home that are not so open to having animals around. The size of the rooms and the laying of the communal areas played a significant role in allowing pets in the homes. One of the managers said that they accept pets because "the homes/flats/rooms belong to people, and the damages caused are usually rectifiable", so that way other residents can engage with the animal if they choose to. Further, having rooms across different floors could help with managing animals in the care home, with one participant saying, "it's just a one storey building, one floor...so it would be hard to keep something like a dog or a cat away." Another care home has rejected animals living with a resident, as there was someone else on the floor who was scared of dogs. Another participant talked about the rooms within the home being quite small and that was mentioned as reason for not letting pets in, as the animals cannot be completely kept away from other residents.

\section{Risk Assessments and Procedures}

We also tried to find out more about any policies or limitations for visiting animals. All of the care homes stated they do have visiting animals, whether this is by resident's families bringing their pets (or the resident's old pets) or by local charities and organisations that specialise in providing animal experiences for the elderly.

The first thing we found was that most of the care homes did not have a particular procedure or any guidance on visiting pets. This meant that essentially, any pets or animals could visit with very little limitations. There were no risk assessment guidelines to follow and when asked, none of them seemed too concerned by this, but most did do some kind of risk assessment. This was something that is strange considering how strict some of the homes were about pets living in the home they seemed quite relaxed about having animals in.

Having said this, it could be down to the fact that the managers acknowledged the fact that they don't have the responsibility of the animals when they are visiting, the responsibility would lay with the families or charities bringing the animals in, and this could be why they don't feel like it is necessary to have a clear procedure for the animals. However, one did say that they "have a risk assessment in place because we have got a lady who is really scared of 
animals", showing acknowledgement for the preferences of the residents. The care home had a two-storey structure and the lady was "ruled out of that floor at the moment".

\section{Opening the Floodgates}

One participant had an interesting perspective on having a set pet policy. They were concerned that if they had a pet policy stating pets were allowed that this would "open the floodgates" and the homes would have as many animal residents as they would human residents. Some of the care homes we spoke to have up to 47 rooms in their care homes, so if they allowed every resident to have a pet, this could potentially be a lot of animals on the premises at one time.

This is something that might be able to be controlled for by allowing for pet policies to be tailored to the home. Whether this is related to the type of animals that are allowed or the number of animals allowed in the home at any one time. Providing this variety will make it easier for residents choosing a care home, as they will now cater for all preferences and also for the care homes to manage the animals that come in to the home.

\section{$\underline{\text { Law }}$}

There was also the issue of the law relating to this matter, or noticeably a lack of legal knowledge on the legality of animals in care homes. From the literature used in our background research, it is clear that there actually isn't a lot of substantive law out there on the area and, therefore, we were not surprised by this lack of knowledge displayed by the managers. This is a large part of the problem, if there is no real guidance on the legal matters as well as the legal consequences, how are the managers meant to know what to do and the legality of their actions?

\section{External Factors}

We also asked participants about external factors such as their tenancy agreements and any issues regarding their landlord or leases. All the care homes stated they were not aware of any restriction on their leases or deeds which limits the amount of animals they can have on the property. Again, it seems to be the individual homes and their owners making the decision on pets in the homes not wider external factors.

\section{Banning 'No Pet' Clauses}

With regards to the potential option favoured by Scottish Labour, of making the use of no pet clauses illegal, all of the managers stated this would be unfair and they believed that the individual care homes, or the care home companies should have the right to decide their own pet policies based on the practicalities and personal preferences. One stated, "it should be up to the individual place... what suits the house and the people that are living in it", which sums up the views of the managers well. Another stated, "I cannot speak for that personally, it would have to be a decision made by the company. There would be a meeting with senior management team and there would be a policy formed to say how the company would respond to that". Another participant emphasised that a blanket ban is not suitable for care homes, stating: 
"People from outside making that decision that pets are allowed in, they don't know the building, they don't know the client that is in there, so they cannot make that decision on a whole basis. It's gotta be at that time and what's best for the building at that time. I don't think anyone should have a no-pet policy, I think they should have to make that decision at the time and whether it's around the clients that they've got in the home."

All the homes, however, did agree that there should be more transparency with their pet policies and allow for more knowledge and choice to be available for prospective residents to allow them to make a better-informed decision.

\section{Choice}

One issue that was frequently mentioned, as discussed above, was the negative impact that animals living in the care home could have on particular residents who were at risk of being distressed by their presence. Concern about residents' choice was more apparent in relation to animals living-in rather than visiting animals, as it has more of an impact on the residents. These concerns often related to space and the challenge of restricting the movements of animals within the home.

\section{Choice of Interaction with Animals}

None of the homes had a communal animal that lived within the house. One of the managers spoke about thinking of getting a dog for the house, but he mentioned such plan was not feasible, as the animal could not stay at the home overnight for more than three nights a week and transportation was not really practical. Another mentioned a care home they worked at previously which had a communal animal:

"years ago I did work in a place, we are talking years ago like 25 years ago, but I was quite shocked when I went there and there was a dog, there was a daschuand and it was enormous, bless it. Everyone just kept...it got all the tip bits, all the leftovers so it was really chunk. And when I got there, there was a bit of an issue with the garden and who was going to clean up the muck and stuff like that and that was a whole thing."

It seems that even when a care home has a communal animal, this brings its own difficulties. Further, the managers did not seem to have any knowledge of s.9 of the Animal Welfare Act 2006 , so possibly would not realise that there is this duty to ensure welfare of the animal.

Other spoke about preferring visiting pets, or pets that could live outside, so that the residents could have some contact and responsibility, without the risks attached with the animal being inside. For example, a participant stated:

"Like chickens...or something like that. I think that would be a bit of an interesting, so that residents, those who are able, could...could be proactive in keeping an eye on them and make sure they are looked after. Or if they weren't in to that, Just as a point of interest... that there is something there 
erm. But me personally, I would be more inclined...to...something outside than something inside."

Again, it is about giving the residents the choice of how much they engage and interact with animals and being able to distance themselves from that if they want to. This was addressed especially in the Ohio University research where, they suggested ways to maximise benefits and reduce risk in facilities by taking simple steps such as, choosing the best animal, as they suggested older and more mature animals can reduce some of the risks such as scratches or bites. The next recommended step is to ensure safe interactions between people and animals, require evidence of proper animal care, and create animal safety and cleanliness protocols for the facility. ${ }^{98}$

\section{Choice of Pet Friendly Care Homes}

All the participants talked about how important it is for the elderly to have the option of moving into a home that would accommodate their needs, such as providing for their pets, but they also mentioned that considering the number of residents, the care home and its manager in particular, should take all those needs of all the residents into account and decide whether an animal is going to be accepted by all the residents in the home.

Most participants stated that they would support a choice of a care home with animals and ones without, one stating:

"I agree that there should be all those choices out there. And that if every care home did something a little bit different and when people were looking they would think, yeah that's the one for me...because it does that thing I like...so.... if they have pets and somebody is really in to that then it's great for them and if someone doesn't, then they can look for somewhere that doesn't do that...so it should be a choice."

This same participant highlighted the reason for this is because they think more people have dogs now than they used to, so this is going to become a bigger issue in the future. Ultimately, it was about being able to choose a home environment which is suitable for the elderly person, whether or not they want to be around animals, and that "they should have that right a well to go to a home that environment is available."

Some said if a resident chooses to give up their animals and move into the home - on which two cases were mentioned - if it was practical, they ask relatives to take the animal in. If this is not possible, they would help the resident to rehome the animal or would ask relevant charities to help them look after and rehome the animal. The managers sometimes weren't sure where the animal had been rehomed to, even though the resident had asked about the dog. It seems, from our data, that care homes will actively help residents, existing or potential, to rehome their animals, particularly in a way that they can still see them. There are some, however, who have such a blanket 'no pet' policy, that the choice is to give up the pet, however that happens, or to find alternative living arrangement.

\footnotetext{
${ }^{98}$ Ohio State University, Model Animal Protocols for Long-Term Care Facilities, <https://kb.osu.edu/bitstream/handle/1811/79017/Model Animal Protocols for LTCF.pdf?sequence=10\&isA llowed $=y>$ accessed on $26^{\text {th }}$ April 2019
} 


\section{Recommendations for Reform}

Managers do recognise some of the benefits that the elderly can receive from a pet. However, this knowledge is very limited and only scratches the surface of the true potential that pets can offer and we think that there could be more education provided for care homes around the need for elderly residents to be housed with their companion animals. Therefore, as a recommendation, it is suggested that there should be more information available for the care homes that teaches the managers of the potential benefits for the elderly with having a pet around. This approach was already implemented by the SCAS 'Pets for Life Campaign'. ${ }^{99}$ Only one participant seemed to have done research around the benefits of pets and, whilst the other participants appreciated this benefit, they did not all seem fully aware of the importance. As stated in the Rowntree Foundation report, the lack of knowledge about the benefits is one of the reasons for having a 'no pet' policy. ${ }^{100}$ It will be useful for care homes to know of this benefit in more detail and be provided with guidance and support of how to accommodate these needs. Further, it may be useful to provide them with detailed guidance on the Animal Welfare Act 2006, specifically s.9, and what duties are placed upon them should they accept an animal into the home.

It was difficult for us to say with confidence which care home operated best, in terms of their pet policies. All care home managers appreciated the importance of animals for some elderly people and did try to cater for this. The position, however is much more difficult than simply allowing pets to enter a care home. The ability to do this depend on staffing, the elderly persons and their needs, the needs of other residents and the layout of the home. Due to this, we do not think it would be appropriate to enforce care homes to allow in all pets, but there does need to be more regulation in place. The decision of whether to allow pets was dependant on the manager, regardless of the care home pet policy, so it could be a luck of the draw situation, as to whether a companion animal can accompany their own into the care home. There did not seem to be any further regulatory or statutory duties on these care homes and, even if they state in their pet policy that they allowed pets, if the manager did not want this it was not allowed. Even if the care home did have a pet policy which allowed resident to bring their pets in, this did not happen if the care home manager was against it. There needs to be more consistency between approaches of care homes and it seems that the only way to provide this is through some kind of statutory procedures and guidance for best practice. Further, there needs to be more information for the residents as to whether or not pets are allowed and, if so, what kinds of animals are allowed. We found that even where care homes did allow pets, there were no residents with pets at the time of the interview. Perhaps if there was better signposting to care homes which allow pets, more elderly people will be able to continue living with their companion animals.

We think that the Care Homes and Sheltered Accommodation (Domestic Pets) Bill was suitable and that it should be reintroduced. When studying s1(2) of the Bill, and when a care home manager can refuse permission for a resident to keep a domestic pet, it is

\footnotetext{
${ }^{99}$ Society for Companion Animal 'Pets For Life Campaign' (2013) <http://www.scas.org.uk/human-animalbond/pets-and-older-people/pets-for-life-campaign/> accessed $29^{\text {th }}$ March 2019

100 Society for Companion Animal Studies, 'Pets And Older People In Residential Care' (2008) <http://176.32.230.19/scas.org.uk/wp-content/uploads/2013/03/3.Pets_and_older_people_report.pdf> accessed $3^{\text {rd }}$ December 2018.
} 
recommended that this include where the layout of the care home is not suitable for certain animals, such as dogs, due to a lack of outside space and small individual rooms. This will be consistent with what the participants were highlighting, particularly that it should be the choice of a home as to whether they allow companion animals into it. Further, we like the idea one of the participants presented, that their care home runs on a democracy and the residents should also be able to have a say in which animals are brought into their home. It may be difficult in practice, but we recommend that local authorities and care homes work together, to perhaps provide homes which are pet friendly, allowing animals to live in with their owners, or give the elderly the choice of moving into a care home which does not have pets. The participants seemed to prefer this option, as it caters for the elderly, but does not put unreasonable obligations on some care homes where it is not suitable for an animal to live. 


\section{Conclusion}

The research team are confident that the research aims of this project were met. For ease of the reader, the research aims were to identify:

- The prevalence of difficulties faced by the elderly population within Newcastle upon Tyne to find accommodation (whether sheltered or in care homes) with their companion animal;

- The reasons why elderly people with companion animals are unable to access accommodation with their companion animals and whether this is due to legal and/or regulatory factors or a perception of the same or other reasons.

- Any steps that could be taken to reduce the obstacles faced by elderly owners of companion animals who are entering the care system or need for sheltered accommodation.

We discovered the difficulties care home managers face with housing the elderly population with their pets in Newcastle upon Tyne and have explored these in some detail with the participants. The findings are, in some ways, consistent with previous studies, but, in other ways, original for the subject area.

We understand that there were some limitations to this study, particularly the small sample size. We are not claiming, however, that this study provide results that can be generalised or that it provides a complete overview of the issues in this area. What we would like to encourage is others to do similar studies in this area, to compare the results with ours, and provide a larger study across England.

Overall, we have found that the reasons for care homes allowing, or not allowing, pets is not black and white. There are various reasons, such as the layout of the home or staffing issues, which can prevent or make care homes reluctant to take companion animals into the home with their owner. We are reluctant to say that legislation should be introduced which will result in all care homes having this requirement, but encourage this to be explored further, ensuring that guidance is in place to support care home managers. 


\section{Appendices}

\section{Appendix 1}

\section{Information for Participants}

Amy Millross, Ellen Martin, Golora Bozorg and Marija Bilerte are full-time students in the Policy Clinic, Student Law Office at Northumbria University. We are undertaking this research for the UK Centre for Animal Law (ALAW). The aim is to explore the Human-Animal relationship between the elderly and their companion animals and we are exploring the difficulties the elderly face to find supported accommodation / care homes that with their companion animals, as well as finding out if there are any reasons why they are unable to stay with their companion animals institutionally. This document sets out the background to the study as a whole and provides detailed information about the part of the study in which you are invited to participate. If there are any questions that are not answered here, please contact us or our supervisor for further information at:

\begin{tabular}{|l|l|}
\hline Student Law Office, & Dr Rachel Dunn (Supervisor) \\
Northumbria School of Law, & Northumbria School of Law, \\
01912273909 & 01912273311 \\
SLO1113@nortumbria.ac.uk & rachel2.dunn@northumbria.ac.uk \\
\hline
\end{tabular}

The issues of housing the elderly with their companion animals

The purpose of this study is to find out two things. Firstly we want to gain an understanding of the views of residents of care homes / supported accommodation on companion animals and collect any experiences or stories to do with their companion animals being accepted or denied into the accommodation. Secondly, we want to find out the similarities and differences of the care homes or supported accommodation with regards to pet policies and how they are enforced and regulated. This research will contribute to the debate about whether or not the law regarding pet ownership in supported care is in need of reform. We will provide this data through a selection of methods:

- A review of literature on the matter of Human-Animal relationships and the benefits of pets for their owners.

- A comparative review of different law and regulations on pet ownership in similar situations across different jurisdictions.

- Interviews with the Managers and/or care-workers of the facilities to find out more about individual pet policies and reasons for allowing or not allowing pets (using audio recording)

\section{What being involved in the research will mean?}

Your participation in this study is voluntary. After signing this form you can withdraw from the study at any time and you will not be asked any questions as to why you no longer wish to take part. If you do withdraw from the study, you may request to re-join at any time; it is at our discretion if you are able to participate again. 
Participation in this study means you provide us with permission to use the data we collect in the group interviews or interviews in the research project and any subsequent publications, by Northumbria University Law School and/or ALAW. Your responses will be combined with that of other participants during the analysis of the responses. Information about individuals or any personal data will not be used in any published reports.

\section{Risks and Benefits of being in the study}

There is no risk in participating in this study, however we are going to be discussing a topic that could potentially cause some upset to some participants. If at any point you feel upset by discussing the matters, you have the right to stop the discussion.

Your participation and opinions may prompt new perspectives and ideas which may be beneficial in the future. If you do not receive a direct benefit from your participation in this study, others may ultimately benefit from the knowledge obtained.

\section{Confidentiality}

The records of this study will be kept confidential to the extent of the General Data Protection Regulation (GDPR) and the Data Protection Act 2018. In any reports on the study, we will not include any personal information that will make it possible to identify any individual or group (for example, participants will be given a letter - Participant A - to not be identifiable). If an individual can be identified by the nature of their role, specific consent to waive confidentiality will be agreed and all written material will be subject to their scrutiny before publication. The audio data collected will not be included within the published research, only the information gained from them. This may include words you have said.

Electronic copies of data, including the audio, will be stored within a secured file, which can only be accessed by the members of the research team, on a password-protected computer in the Student Law Office. The file is stored on a secure drive that cannot be accessed outside of the office. Extracts may be shared and viewed by the research team and the organisation instructing the research, and publications will be generated based on the data. All documentation will be made anonymous prior to this to maintain participant confidentiality. A copy of the data will also be stored on the Supervisor's password-protected computer, in a locked office.

The audio recordings on the Dictaphone will be deleted within two weeks of the initial recording. The saved audio recordings will be deleted from the Student Law Office university computers after the research project has finished. However, electronic copies will be retained by the Supervisor for up to 1 year any electronic and hard copies will be held for a period of up to 6 years in the Student Law Office. Within this time you may request to see any data collected in this particular study. However, as stated above, all names of individuals will not be used and supported accommodation/care home facilities will be given a letter instead of their usual name.

\section{Consent to Participate in the Element of the Study}

You will be participating in an interview. You will be participating in face-to-face interviews (telephone interviews where face-to-face is not practical or feasible). We will use a Dictaphone to capture your voice to enable us to make transcripts of your comments. Interviews are expected to last around 30-60 minutes.

I have read and understand the study that I will be participating in;

I have been given an opportunity to ask questions about the study; 
I understand that taking part in the study will include being audio recorded;

I have been given adequate time to consider my decision to participate;

I understand that any of my personal details, such as my name, will not be revealed to anyone outside of the research team;

I understand that my words may be quoted in publications, reports and other research outputs but my name will not be used;

$\square$ I understand I can withdraw from the study at any time and I will not be asked any questions about why I no longer wish to take part.

I agree to participate in the study;

I agree to the University of Northumbria University at Newcastle recording and processing this information about me. I understand that this information will be used only for the purpose(s) set out in this information sheet supplied to me, and my consent is conditional upon the university complying with its duties obligations under the Data Protection Act 2018.

Name of Participant:

Date:

Name of Researcher:

Date: 


\section{Appendix 2}

Dear (Managers name),

We hope this email finds you well.

We are Marija Bilerte, Amy Millross, Golara Bozorg and Ellen Martin, students from Northumbria University's Student Law Office, working alongside UK Centre for Animal Law (ALAW) on a research project entitled 'The difficulty for the elderly population and companion animals to remain living together in supported accommodation/care homes'. We are supervised by Dr Rachel Dunn on this project.

The purpose of the research is to:

- recognise the difficulty that elderly people face finding accommodation with their companion animal;

- reasons why the elderly may be unable to access accommodation with their companion animal;

- identifying organisations which allow this and their practices and procedures;

- and identifying any steps that could be taken to reduce obstacles faced by the elderly who are entering the care system.

There are many benefits of elderly persons living with their companion animals. So far, however, we have discovered there is often a problem of the elderly having to rehome their companion animals, as some supported accommodations do not allow pets as part of their policies.

We are inviting care home managers and residents to participate in interviews to discuss their views on housing the elderly with their companion animals in supported accommodation/care homes and any reasons why you would believe there is a problem with housing the elderly with pets. The information collected will be used to help us explain what the problems you may be facing and what guidance and/or legislation could be put in place to help the elderly be housed with their companion animals. The interview could take place at your supported accommodation/care home or over the phone. We would like to know if you would be able to express your views on this subject.

If you have any questions about the project then please contact us. You can contact us via email at la.studentlawoffice@northumbria.ac.uk or our supervisor at rachel2.dunn@northumbria.ac.uk and we will be happy to discuss any questions you may have.

Yours Faithfully,

Marija Bilerte, Amy Millross and Golara Bozorg

Student Advisors

Northumbria Student Law Office 


\section{Appendix 3}

1. Do you allow pets in your accommodation?

IF YES:

1. Does the setting have a policy or guidance about keeping pets in the accommodation?

- If yes, what is it? What's the procedure?

- Where does the guidance come from? Was it made internally or are you using an external source?

- What sort of issues are covered in the guidance? (ASK FOR MATERIALS)

- Are you aware of (or do you refer to) guidance or information published by any of the companion animal charities, such as RSPCA, PDSA etc...

2. Have you found there to be benefits to residents in the setting of having pets?

- If yes, what benefits?

- Do you think they are important for the care home residents?

3. Have you experienced any problems with having pets in the setting, in terms of noise, mess, damage to property and/ or health?

- If yes, what are the problems and how do you overcome them in the setting?

- Where are they allowed in the setting?

- Who is responsible for feeding, exercising and cleaning up after pets?

4. What happens to a pet if the owner dies?

5. Do you charge an increased rent for those with pets?

6. Do you have on-site / accessible vets?

- Who bears the cost of this?

IF NO:

1. Do you have any reasons for not allowing pets?

- What internal information or guidance do you rely upon?

- Are there any external factors that you are aware of prohibiting pets in the setting?

2. Would you ever consider allowing pets?

- If yes, what would make you consider this?

- If no, what is the biggest factor for not considering it?

LEGAL:

1. What knowledge do you have on the law surrounding pets in supported accommodation?

2. Would you like there to be more guidance in the law on this matter?

- Do you think there should be legislation on this area of law? 
- How would you feel if 'no pet' clauses were made illegal?

3. If legislation was passed, what would you want the law to cover?

- And what would you not like to be included in it? 


\section{Appendix 4}

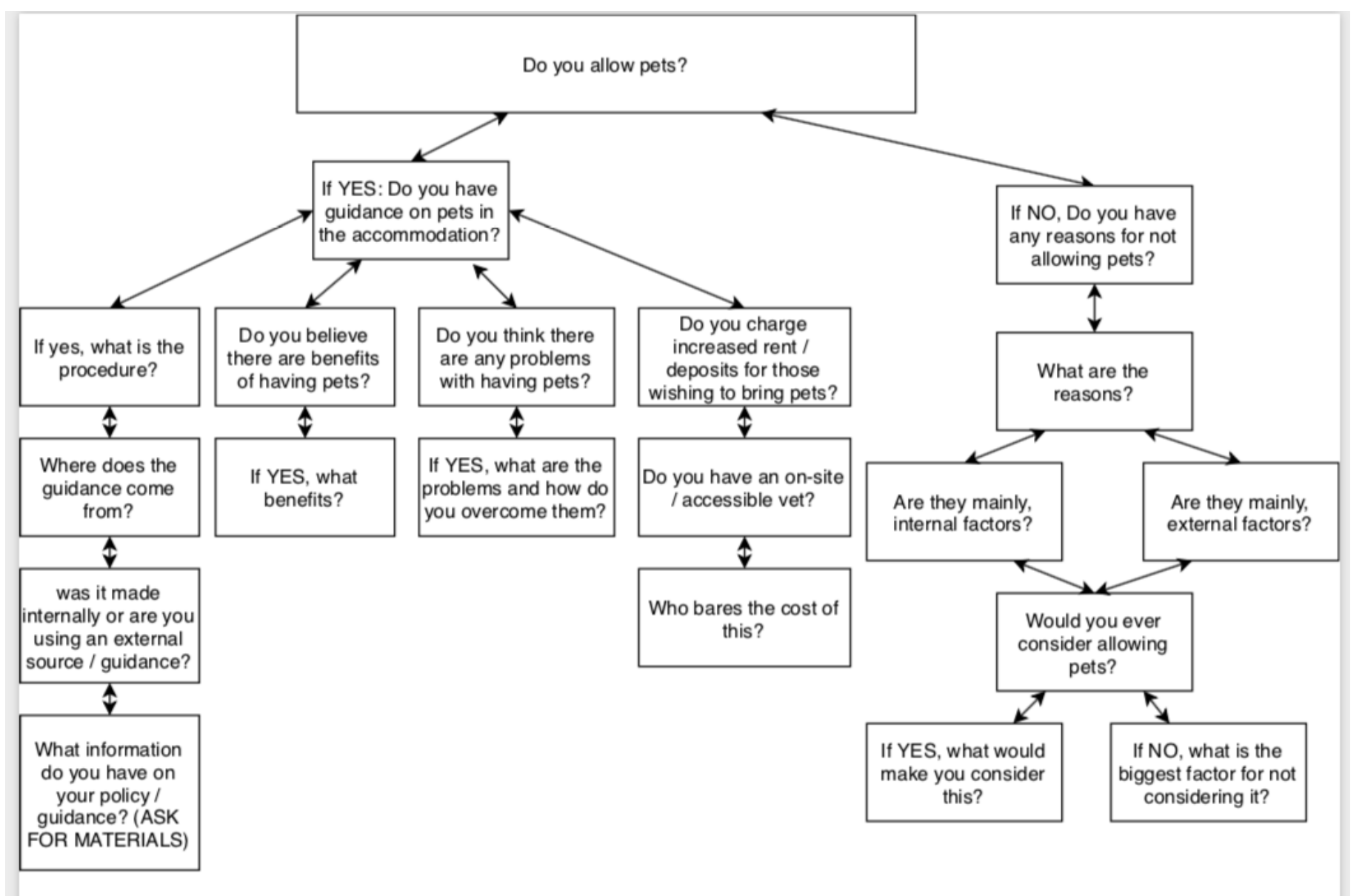

